\title{
Reprogramming and genome integrity: role of non-homologous end joining
}

\author{
P Salomoni ${ }^{*}, 1$ \\ Cell Death and Differentiation (2013) 20, 1285-1286; doi:10.1038/cdd.2013.95
}

The study by Tilgner et al. ${ }^{1}$ investigates the role of nonhomologous end joining (NHEJ) in reprogramming of human somatic cells to induced pluripotent stem cells and in regulation of their differentiation. NHEJ is the major double-strand break (DSB) repair pathway in mammalian cells. ${ }^{2}$ It is a highly coordinated process relying on a number of essential proteins, such as DNA ligase IV, which is required for the final 'end processing' step. ${ }^{3}$ Mutations in DNA ligase IV lead to the LIG4 syndrome characterised by growth defects, microcephaly, impaired haematopoiesis, immunodeficiency and increased susceptibility to leukemia. Complete loss of DNA ligase IV in the mouse leads to embryonic lethality, suggesting that mutations in the LIG4 syndrome are generally hypomorphic.

Defects in NHEJ have been shown to affect stem cell self-renewal and differentiation in the haematopoietic system, suggesting that endogenous DNA damage needs to be kept to the minimum during haematopoiesis. What about pluripotent stem cells? Although mouse embryonic stem cells appear to rely mainly on homologous recombination (HR), both $\mathrm{HR}$ and NHEJ have been proposed to function in human ESCs as well as induced pluripotent stem cells (iPSCs). iPSC reprogramming has emerged as a key technology to study molecular mechanisms underlying human hereditary syndromes, with implications for drug development as well as regenerative medicine. This study aims to define the impact of either NHEJ or HR deficiencies on generation of iPSCs and their differentiation.

Tilgner et al. ${ }^{1}$ have created three iPSC lines from LIG4 patient-derived fibroblasts using conventional reprogramming protocols based on OCT4, SOX2, KLF4 and C-MYC transduction. Although the authors were able to obtain iPSC lines from control and LIG4 fibroblasts, the reprogramming efficiency was much reduced when DNA ligase IV was mutated. Although p53 has been shown to rescue some of the phenotypes caused by DNA ligase IV loss in the mouse, ${ }^{4}$ p53 downregulation did not have any effect on the reprogramming defect observed in the absence of DNA ligase IV. The limited reprogramming capacity was associated with increased genomic instability, which was rescued by reintroduction of DNA ligase IV. Notably, DNA ligase IV-deficient iPSCs were impaired in teratoma formation capacity, suggesting a differentiation defect upon impairment of NHEJ. These phenotypes were associated with impaired repair of ionising irradiation (IR)-induced DSBs as well as increased DSB foci at steady state. Together, these data clearly show that NHEJ is required for efficient DNA repair during iPSC reprogramming as well as in established iPSCs.

A number of questions arise from these interesting findings. First, it would be important to determine whether iPSC reprogramming induces DNA damage. It is conceivable that reprogramming factors could induce DNA damage potentially via replication stress associated with increased proliferative index. In this respect, reprogramming of somatic mouse cells results in replication stress, which is in part dependent on c-Myc. $^{5}$ This would explain the positive effect of p53 loss on reprogramming efficiency. ${ }^{6}$ But why this effect is not observed in the context of DNA ligase IV inhibition (this study)? One could speculate that cell death induced by impaired DNA repair in DNA ligase IV-deficient cells is at least in part p53 independent. In this respect, p53 loss would increase survival in the presence of an intact DNA repair machinery, but levels of DNA damage would be too high when NHEJ is impaired, thus potentially activating forms of cell death that do not rely on p53. This would be in agreement with previous findings showing that the radiosensitivity phenotype of DNA ligase IV-deficient mice is not rescued by p53 loss. ${ }^{4}$ In contrast, in established LIG4-iPSCs p53 knockdown clearly inhibits cell death at both steady state and upon IR. Interestingly, this type of cell death seems to rely on p53-mediated activation of the Rho-associated kinase (ROCK) and ROCK-dependent compaction of the actin network. Indeed, pharmacologically inhibition of ROCK or actin compaction limits cell death in LIG4 iPSCs. ROCK inhibitors could represent a novel strategy to improve viability of iPSC cultures.

The authors then investigated the effect of NHEJ deficiency on haematopoietic specification. Treatment with BMP4, bFGF and VEGF led to the emergence of haemato-endothelial progenitors, primitive and total blood cells. Although haematopoietic specification appeared normal in LIG4 iPSC cultures, LIG4 cells generated a higher number of haemato-endothelial progenitors. Interestingly, this effect was abolished by p53 knockdown, suggesting that the p53/ROCK pathway is required for enhancing haematopoietic differentiation, as recently reported by the same group. ${ }^{7}$ Analysis of differentiation using colony-forming unit (CFU) assays revealed a significant reduction of LIG4 cells to form haematopoietic

\footnotetext{
${ }^{1}$ Samantha Dickson Brain Cancer Unit, UCL Cancer Institute, Paul O'Gorman Building, 72 Huntley Street, London WC1H 6BT, UK

*Corresponding author: P Salomoni, Samantha Dickson Brain Cancer Unit, UCL Cancer Institute, Paul O'Gorman Building, 72 Huntley Street, London WC1H 6BT, UK. Tel: + 440207679 0728; Fax: + 440207679 6643; E-mail: p.salomoni@cancer.ucl.ac.uk
} 


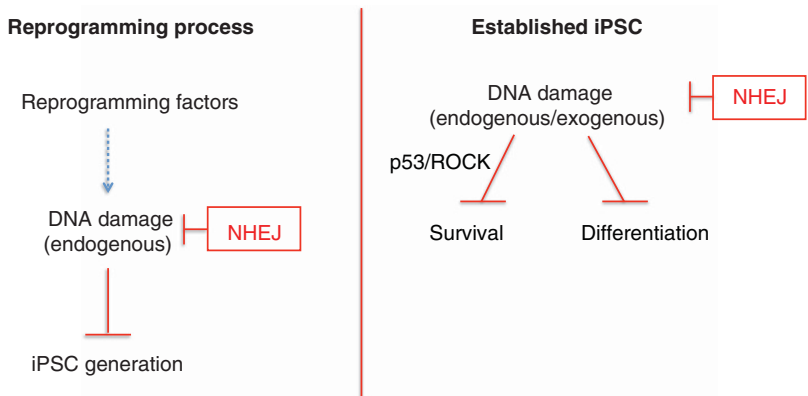

Figure 1 Role of NHEJ in human-induced pluripotent stem cells (iPSCs). Tansduction of somatic cells with reprogramming factors is likely inducing DNA damage in addition to epigenetic/transcriptional reprogramming. In this context, NHEJ limits DNA damage, thus increasing reprogramming efficiency (left panel). In established IPSCs, NHEJ mediates repair of both endogenous and exogenous DNA damage, thus sustaining haematopoietic differentiation (right panel). Furthermore, NHEJ-mediated DNA repair suppressesinduction of a p53/ROCK non-canonical cell death pathway

colonies, which inversely correlated with residual DNA ligase IV activity. This correlation is corroborated by clinical data suggesting an increased severity of the haematopoietic phenotype in patients with the least residual ligase activity. Interestingly, although DNA ligase IV expression rescued the phenotype, p53 knockdown failed to do the same, suggesting that the CFU defect is not dependent on p53 and may rely on p53-independent cell death. When the authors attempted to correlate the haematopoietic phenotype with levels of DNA damage, they found that in differentiating LIG4 cultures the
CD34 + CD45 + primitive subpopulation is most affected with respect to DSBs and apoptosis. Rather counter intuitively, the same subpopulation also showed increased proliferation (see above). As the authors hypothesised, increased cell death in this compartment could act as a positive feedback loop for augmented production of haemato-endothelial progenitors. Accordingly, in the absence of p53 this increase was not observed. Finally, DNA damage in non-haematopoietic lineages did not seem to be increased upon NHEJ impairment, suggesting that compensatory HR is activated. It is unclear whether the authors have also investigated specification towards the neural lineages, especially in view of the central nervous system phenotype of NHEJ deficiency.

Overall, this study provides important insights into the role of NHEJ in regulation of genomic stability during iPSC reprogramming as well as in established iPSCs (Figure 1). This could lead to the development of new protocols for improving viability and genomic stability during in vitro expansion of iPSCs. More broadly, this work improves our understanding of the interplay between the DNA damage repair machinery and cell fate regulation in pluripotent stem cells.

1. Tilgner $\mathrm{K}$ et al. Cell death and differ 2013; 20: 1089-1100.

2. Lieber MR, Ma Y, Pannicke U, Schwarz K. Nat Rev Mol Cell Biol 2003; 4: 712-720.

3. Ahnesorg P, Smith P, Jackson SP. Cell 2006; 124: 301-313.

4. Frank KM et al. Mol Cell 2000; 5: 993-1002.

5. Pasi CE et al. Cell Death Differ 2011; 18: 745-753.

6. Marion RM et al. Nature 2009; 460: 1149-1153.

7. Yung S et al. Hum Mol Genetics 2011; 20: 4932-4946. 